\title{
Uniform stability and semi-stability of motions in dynamical systems on metric spaces
}

\author{
by Andrzej Pelczar (Kraków)
}

\begin{abstract}
Some stability properties of motions in pseudo-dynamical systems and semi-systems are studied.

Introduction. The purpose of the present paper is to study some properties of motions in dynamical and pseudo-dynamical systems (see definitions below) on metric spaces. We consider uniform stability and semistability (see Section 2) of motions in nonempty subsets of the phase space and discuss in particular properties of mappings of the types:

$$
x \longmapsto\{\text { the limit set of } x\}
$$

and

$$
x \longmapsto\{\text { the prolongational limit set of } x\},
$$

as well as some regularity properties of mappings whose values are some generalized prolongational limit sets (see the formula (1.8)). In Sections 3 and 4 we consider the problem of propagation of stability and uniform stability of motions, from sets onto closures of those sets.

In Section 7 we consider certain Lyapunov type functions.

The paper is closed by some remarks concerning stability properties of motions preserved when we pass from one dynamical system to another, assuming that those systems satisfy certain conditions of the type of "asymptotic equivalence" in the Ważewski sense.
\end{abstract}

1. Preliminaries. In order to exclude any misunderstanding we recall fundamental definitions and fix notation used in the sequel.

1991 Mathematics Subject Classification: 34C35, 54H20, 58F10, 58F25, 34D05, 34D20.

Key words and phrases: stability, semi-stability, limit set, prolongational limit set, generalized prolongational limit set, asymptotic equivalence.

The paper was supported by Polish Scientific Grant 210779101. 
Let $(X, \varrho)$ be a metric space (fixed throughout the paper) and let $\pi$ be a mapping from $\mathbb{R} \times X$ (resp. from $\mathbb{R}_{+} \times X$, where $\mathbb{R}_{+}$is the additive semi-group of nonnegative real numbers) into $X$. We say that the triple $(X, \mathbb{R} ; \pi)$ (resp. $\left.\left(X, \mathbb{R}_{+} ; \pi\right)\right)$ is a pseudo-dynamical system (a pseudo-dynamical semi-system) if:

(i) $\pi(0, x)=x$ for every $x \in X$,

(ii) $\pi(t, \pi(s, x))=\pi(t+s, x)$ for $x \in X$ and $t, s \in \mathbb{R}$ (resp. $t, s \in \mathbb{R}_{+}$).

If $\pi$ is continuous with respect to the product topology in $\mathbb{R} \times X$ (resp. $\left.\mathbb{R}_{+} \times X\right)$, then the triple $(X, \mathbb{R} ; \pi)$ (the triple $\left.\left(X, \mathbb{R}_{+} ; \pi\right)\right)$ is said to be a dynamical system (a dynamical semi-system).

For any fixed $t \in \mathbb{R}$ (resp. $t \in \mathbb{R}_{+}$) we define the $t$-translation by

$$
\pi_{t}: X \ni x \mapsto \pi_{t}(x):=\pi(t, x) \in X .
$$

For any fixed $x \in X$ we define the motion of $x$ by

$$
\begin{gathered}
\pi^{x}: \mathbb{R} \ni t \mapsto \pi^{x}(t):=\pi(t, x) \in X \\
\text { (resp. } \left.\pi^{x}: \mathbb{R}_{+} \ni t \mapsto \pi(t, x) \in X\right) .
\end{gathered}
$$

If $(X, \mathbb{R} ; \pi)$ is a pseudo-dynamical system (resp. $\left(X, \mathbb{R}_{+} ; \pi\right)$ is a pseudodynamical semi-system) then for $x \in X$ we denote by $\pi(x)$ the set

$$
\{\pi(t, x): t \in \mathbb{R}\} \quad\left(\text { resp. }\left\{\pi(t, x): t \in \mathbb{R}_{+}\right\}\right)
$$

and we call it the trajectory of $x$; for the system $(X, \mathbb{R} ; \pi)$, we also consider the sets

$$
\pi_{+}(x):=\{\pi(t, x): t \geq 0\}, \quad \pi_{-}(x):=\{\pi(t, x): t \leq 0\}
$$

called the positive and negative semi-trajectories of $x$.

R e mark 1.0. Observe that if $(X, \mathbb{R} ; \pi)$ is a pseudo-dynamical (dynamical) system then putting

$$
\pi_{+}:=\left.\pi\right|_{\mathbb{R}_{+} \times X}, \quad \pi_{-}:=\left.\pi\right|_{\mathbb{R}_{-} \times X}
$$

we obtain two natural pseudo-dynamical semi-systems: $\left(X, \mathbb{R}_{+} ; \pi_{+}\right)$and $\left(X, \mathbb{R}_{-} ; \pi_{-}\right)$. It is clear that the trajectory of any point $x \in X$ in the semisystem $\left(X, \mathbb{R}_{+} ; \pi_{+}\right)$(resp. $\left.\left(X, \mathbb{R}_{-} ; \pi_{-}\right)\right)$is equal to the positive (negative) semi-trajectory of the point $x$ in the system $(X, \mathbb{R} ; \pi)$.

From now on, we concentrate on semi-systems (dynamical and pseudodynamical), since corresponding results for systems are obtainable via Remark 1.0 .

Let $\left(X, \mathbb{R}_{+} ; \pi\right)$ be a pseudo-dynamical semi-system. For $x \in X$ we define

$$
\begin{aligned}
\Lambda(x):=\{y \in X: & \text { there is a sequence }\left\{t_{n}\right\} \text { of nonnegative real } \\
& \text { numbers such that } t_{n} \rightarrow \infty \text { and } \pi\left(t_{n}, x\right) \rightarrow y \\
& \text { as } n \rightarrow \infty\},
\end{aligned}
$$




$$
\begin{gathered}
J(x):=\left\{y \in X: \text { there are sequences }\left\{t_{n}\right\} \text { and }\left\{x_{n}\right\}\right. \text { such that } \\
\qquad t_{n} \in \mathbb{R}_{+}, x_{n} \in X, t_{n} \rightarrow \infty, x_{n} \rightarrow x \text { and } \\
\\
\left.\pi\left(t_{n}, x_{n}\right) \rightarrow y \text { as } n \rightarrow \infty\right\},
\end{gathered}
$$

and call them the limit set of $x$ and the prolongational limit set of $x$, respectively (compare for instance [1]-[3]).

Let now $A$ be a nonempty subset of $X$. We put (compare [4])

$$
\begin{aligned}
\widehat{J}(A):=\{y \in X: & \text { there are sequences }\left\{t_{n}\right\} \text { and }\left\{x_{n}\right\} \text { such that } \\
& t_{n} \in \mathbb{R}_{+}, x_{n} \in X, t_{n} \rightarrow \infty, \varrho\left(x_{n}, A\right) \rightarrow 0 \text { and } \\
& \left.\pi\left(t_{n}, x_{n}\right) \rightarrow y \text { as } n \rightarrow \infty\right\} .
\end{aligned}
$$

Here $\varrho(z, A):=\inf \{\varrho(z, y): y \in A\}$.

If $(X, \mathbb{R} ; \pi)$ is a system, then of course we can consider positive and negative limit sets and positive and negative prolongational limit sets defined as limit sets or prolongational limit sets in suitable semi-systems $\left(X, \mathbb{R}_{+} ; \pi_{+}\right)$ and $\left(X, \mathbb{R}_{-} ; \pi_{-}\right)$; they are denoted by $\Lambda^{+}(x), \Lambda^{-}(x), J^{+}(x)$ and $J^{-}(x)$ respectively (see [1]-[3]; compare also Remark 1.0).

Similarly we consider $\widehat{J}^{+}(A)$ and $\widehat{J}^{-}(A)$ (see [4]).

A motion $\pi^{x}$ is said to be Lyapunov stable (see for instance [1]) if and only if for every $\varepsilon>0$ there is $\delta>0$ such that

$$
\varrho(x, y)<\delta \Rightarrow\{\varrho(\pi(t, x), \pi(t, y))<\varepsilon \text { for } t \geq 0\} .
$$

In [2] a weaker condition was introduced.

Let $x \in X$. We say that the motion $\pi^{x}$ is semi-stable if for every $\varepsilon>0$ there are $\delta>0$ and $s \geq 0$ such that

$$
\varrho(x, y)<\delta \Rightarrow\{\varrho(\pi(t, x), \pi(t, y))<\varepsilon \text { for } t \geq s\} .
$$

For systems we can of course consider positive and negative stability and semi-stability (see [1], [4]).

Remark 1.1. If $\pi$ is continuous in $\{x\} \times \mathbb{R}_{+}$, then the stability of the motion $\pi^{x}$ is equivalent to its semi-stability.

We now introduce some sets useful in the sequel; in their definitions depending on a given subset $E$ of $X$ and $t \in \mathbb{R}_{+}$, we need the restriction

$$
\left.\pi_{t}\right|_{E \cup\{x\}}: E \cup\{x\} \ni y \mapsto \pi(t, y) \in X
$$

for $x \in \partial E$.

If $E$ is a nonempty subset of $X$, then for $t^{0} \geq 0$ we put

$$
C\left(E ; t^{0}\right):=\left\{x \in \bar{E}: \text { for every } t \geq t^{0}\right. \text { the mapping (1.11) is contin- }
$$
uous at the point $x\}$.

Furthermore, we define

$$
C(E ; *):=\bigcup\left\{C\left(E ; t^{0}\right): t^{0} \geq 0\right\} .
$$


For systems, we consider sets $C_{+}\left(E ; t^{0}\right)$ (with $t^{0} \geq 0$ ) and $C_{-}\left(E ; t^{0}\right)$ (for $\left.t^{0} \leq 0\right)$ as well as $C_{+}(E ; *)$ and $C_{-}(E ; *)$ defined in a natural way.

In [2] a condition of "local uniform compactness" type was introduced. Let $\alpha>0$ be fixed. We say that the condition $\operatorname{Comp}[\alpha]$ is satisfied at a point $x \in X$ iff the ball $B(x, \alpha)=\{y \in X: \varrho(x, y)<\alpha\}$ is relatively compact (i.e. its closure is compact). If $\operatorname{Comp}[\alpha]$ is satisfied at every $x \in E$, then we write briefly $E \in \operatorname{Comp}[\alpha]$.

It is clear that if $(X, \varrho)$ is locally compact then for every compact set $E \neq \emptyset$ there is $\alpha>0$ such that $E \in \operatorname{Comp}[\beta]$ for each $\beta \in(0, \alpha]$.

For $\alpha>0$ and $A \subseteq X, A \neq \emptyset$, we put

(1.14) $\mathcal{L}(A ; \alpha):=\{x \in A: \Lambda(x) \neq \emptyset$ and $\Lambda(x) \in \operatorname{Comp}[\alpha]\}$,

(1.15) $\mathcal{J}(A ; \alpha):=\{x \in A: J(x) \neq \emptyset$ and $J(x) \in \operatorname{Comp}[\alpha]\}$,

(1.16) $\widehat{\mathcal{J}}(A ; \alpha):=\{x \in A: \widehat{J}(\pi(x)) \neq \emptyset$ and $\widehat{J}(\pi(x)) \in \operatorname{Comp}[\alpha]\}$.

For dynamical and semi-dynamical systems we can of course consider sets $\mathcal{L}^{+}(A ; \alpha), \mathcal{L}^{-}(A ; \alpha), \mathcal{J}^{+}(A ; \alpha)$, etc., defined according to the general method based on Remark 1.1.

Denote by $\mathcal{P}(X)$ the family of all nonempty subsets of $X$. We need some semi-continuity conditions of uniform type for mappings from $X$ into $\mathcal{P}(X)$. Let $F: X \rightarrow \mathcal{P}(X)$ and $E \in \mathcal{P}(X)$ be given. We say that $F$ is uniformly upper semi-continuous in $E$ if for every $\varepsilon>0$ there is $\delta>0$ such that

$$
\{x, y \in E, \varrho(x, y)<\delta\} \Rightarrow\{F(x) \subseteq B(F(y), \varepsilon) \text { and } F(y) \subseteq B(F(x), \varepsilon)\} .
$$

Here $B(Z, r):=\{y \in X: \varrho(y, Z)<r\}$ for $Z \in \mathcal{P}(X)$ and $r>0$.

For $C \in \mathcal{P}(X)$ we sometimes write $\pi(t ; C)$ instead of $\pi_{t}(C)$ (especially when $t$ has subscripts).

2. Uniform stability and semi-stability of motions. Let $\left(X, \mathbb{R}_{+} ; \pi\right)$ be a pseudo-dynamical semi-system and let $A \in \mathcal{P}(X)$. If $\pi^{x}$ is stable or semi-stable for every $x \in A$, then we say that $\pi^{A}$ is stable in $X$ or semi-stable in $X$, respectively.

Below we propose a more general terminology. Let $D$ be a subset of $X$ such that $A \subseteq D$. We say that $\pi^{A}$ is stable in $D$ if for every $x \in A$ and every $\varepsilon>0$ there is $\delta>0$ such that

$$
\{y \in D, \varrho(x, y)<\delta\} \Rightarrow\{\varrho(\pi(t, x), \pi(t, y))<\varepsilon \text { for } t \geq 0\} ;
$$

we say that $\pi^{A}$ is semi-stable in $D$ if for every $x \in A$ and each $\varepsilon>0$ there are $\delta>0$ and $s \geq 0$ such that

$$
\{y \in D, \varrho(x, y)<\delta\} \Rightarrow\{\varrho(\pi(t, x), \pi(t, y))<\varepsilon \text { for } t \geq s\} .
$$

Suitable modifications of these definitions (positive and negative stability and semi-stability of $\pi^{A}$ in $D$ ) for systems are obvious. 
Observe that $\pi^{A}$ is stable or semi-stable in $X$ if and only if $\pi^{A}$ is stable or semi-stable, respectively, in some open set $D$ containing $A$. If $\pi^{A}$ is stable or semi-stable in $A$, then we shall say briefly that $\pi^{A}$ is stable or semi-stable, respectively.

Let $B$ be a subset of $X$ such that $A \subseteq B$. We say that $\pi^{A}$ is uniformly stable in $B$ if for every $\varepsilon>0$ there is $\delta>0$ such that

$$
\{x \in A, y \in B, \varrho(x, y)<\delta\} \Rightarrow\{\varrho(\pi(t, x), \pi(t, y))<\varepsilon \text { for } t \geq 0\} .
$$

If $\pi^{A}$ is uniformly stable in $A$, then we say simply that $\pi^{A}$ is uniformly stable.

We say that $\pi^{A}$ is uniformly semi-stable in $B$ if for every $\varepsilon>0$ there are $\delta>0$ and $s \geq 0$ such that

$$
\{x \in A, y \in B, \varrho(x, y)<\delta\} \Rightarrow\{\varrho(\pi(t, x), \pi(t, y))<\varepsilon \text { for } t \geq s\} .
$$

If $\pi^{A}$ is uniformly semi-stable in $A$, then we say that $\pi^{A}$ is uniformly semistable.

"Positive" and "negative" versions of these notions are defined as previously.

R e m ark 2.1. The notion of uniform stability was introduced in [1] (see Def. 6.3); here we use a slightly modified notation.

It is obvious that uniform stability (semi-stability) of $\pi^{A}$ in any set $B$ containing $A$ implies stability (semi-stability) of $\pi^{A}$ in $A$, but not conversely.

Proposition 2.1. If $A$ is compact then semi-stability of $\pi^{A}$ in $D(A \subseteq$ $D \subseteq X)$ implies uniform semi-stability of $\pi^{A}$ in $D$.

An obvious proof will be omitted.

R e mark 2.2. Observe that uniform stability of $\pi^{A}$ in $\bar{A}$ does not imply in general stability of $\pi^{A}$ in $X$; in particular, uniform stability of $\pi^{A}$ does not imply that every motion $\pi^{x}$ with $x \in A$ is stable in the sense of the classical definition mentioned in Section 1.

3. Uniform stability (semi-stability) of $\pi^{A}$ in dynamical semisystems implies uniform stability (semi-stability) of $\pi^{\bar{A}}$. Let $\left(X, \mathbb{R}_{+} ; \pi\right)$ be a pseudo-dynamical semi-system. Let $A \in \mathcal{P}(X)$.

LEMMA 3.1. If $\pi^{A}$ is uniformly semi-stable in $\bar{A}$, then $\pi^{\bar{A}}$ is uniformly semi-stable.

$\operatorname{Proof}$. Let $\varepsilon>0$ be given and let $\delta>0$ and $t^{0} \geq 0$ be chosen in such a way that

$\{x \in A, y \in \bar{A}, \varrho(x, y)<\delta\} \Rightarrow\left\{\varrho(\pi(t, x), \pi(t, y))<\varepsilon / 3\right.$ for $\left.t \geq t^{0}\right\}$ and put $\sigma:=\delta / 2$. Fix $x, y \in \bar{A}$ such that $\varrho(x, y)<\sigma$ and take $u, v \in A$ such 
that $\varrho(x, u)<\delta / 4$ and $\varrho(y, v)<\delta / 4$. Clearly $\varrho(u, v)<\delta$ and so

$$
\varrho(\pi(t, u), \pi(t, v))<\varepsilon / 3 \quad \text { for } t \geq t^{0} .
$$

Also for $t \geq t^{0}$,

(jj) $\quad \varrho(\pi(t, x), \pi(t, u))<\varepsilon / 3 \quad$ and $\varrho(\pi(t, y), \pi(t, v))<\varepsilon / 3$.

Applying the triangle inequality, from $(\mathrm{j})$ and $(\mathrm{jj})$ we get

$$
\varrho(x, y) \leq \varrho(x, u)+\varrho(u, v)+\varrho(v, y) \leq \varepsilon \quad \text { for } t \geq t^{0},
$$

which finishes the proof.

THEOREM 3.1. Assume that there is $t^{0} \geq 0$ such that for every $t \geq t^{0}$ the mapping

$$
\left.\pi_{t}\right|_{\bar{A}}: \bar{A} \ni x \mapsto \pi_{t}(x)=\pi(t, x) \in X
$$

is continuous at every point of $\bar{A}$. If $\pi^{A}$ is uniformly semi-stable, then $\pi^{\bar{A}}$ is also uniformly semi-stable.

Pr o of. Let $\varepsilon>0$ be given. Fix $x \in A$. Since $\pi^{A}$ is uniformly semi-stable, there are $\delta>0$ and $s \geq 0$ such that

$$
\{y, w \in A, \varrho(y, w)<\delta\} \Rightarrow\{\varrho(\pi(t, y), \pi(t, w))<\varepsilon / 2 \text { for } t \geq s\} .
$$

We show that

$$
\begin{aligned}
& \{y \in \bar{A}, \varrho(x, y)<\delta / 2\} \\
& \quad \Rightarrow\left\{\varrho(\pi(t, x), \pi(t, y))<\varepsilon \text { for } t \geq \max \left(t^{0}, s\right)\right\} .
\end{aligned}
$$

Fix $y^{0} \in B(x, \delta / 2) \cap \bar{A}$ and $r \geq \max \left(s, t^{0}\right)$. By assumption $\pi_{r}$ is continuous at $x$ and at $y^{0}$. So there are $\gamma_{1}, \gamma_{2}>0$ such that

$$
\left\{w \in A, \varrho(x, w)<\gamma_{1}\right\} \Rightarrow \varrho(\pi(r, x), \pi(r, w))<\varepsilon / 4
$$

and

$$
\left\{z \in A, \varrho\left(y^{0}, z\right)<\gamma_{2}\right\} \Rightarrow \varrho\left(\pi\left(r, y^{0}\right), \pi(r, z)\right)<\varepsilon / 4 .
$$

We may require $\gamma_{1}$ and $\gamma_{2}$ to be less than $\delta / 4$. Take $w^{0} \in B\left(x, \gamma_{1}\right) \cap A$ and $z^{0} \in B\left(y^{0}, \gamma_{2}\right) \cap A$. It is clear that $\varrho\left(w^{0}, z^{0}\right)<\delta$ and so (see $\left.(3.2)\right)$ we have

$$
\varrho\left(\pi\left(t, z^{0}\right), \pi\left(t, w^{0}\right)\right)<\varepsilon / 2 \quad \text { for } t \geq s,
$$

which gives in particular

$$
\varrho\left(\pi\left(r, z^{0}\right), \pi\left(r, w^{0}\right)\right)<\varepsilon / 2 .
$$

Using the triangle inequality we get (by (3.4)-(3.6))

$$
\begin{aligned}
\varrho\left(\pi(r, x), \pi\left(r, y^{0}\right)\right) \leq & \varrho\left(\pi(r, x), \pi\left(r, z^{0}\right)\right)+\varrho\left(\pi\left(r, z^{0}\right), \pi\left(r, w^{0}\right)\right) \\
& +\varrho\left(\pi\left(r, w^{0}\right), \pi\left(r, y^{0}\right)\right) \\
\leq & \varepsilon / 4+\varepsilon / 2+\varepsilon / 4=\varepsilon,
\end{aligned}
$$

proving (3.3). 
Thus we have proved uniform semi-stability of $\pi^{A}$ in $\bar{A}$. It is now enough to apply Lemma 3.1.

It is easy to see that modifying in a natural way (even: slightly simplifying) the reasoning presented in the proofs of Lemma 3.1 and Theorem 3.1 we can prove the following lemma and theorem:

LEMMA 3.2. If $\pi^{A}$ is uniformly stable in $\bar{A}$, then $\pi^{\bar{A}}$ is uniformly stable.

THEOREM 3.2. Assume that for every $t \geq 0$ the mapping (3.1) is continuous in $\bar{A}$. If $\pi^{A}$ is uniformly stable, then $\pi^{\bar{A}}$ is uniformly stable.

Corollary 3.1. If $\left(X, \mathbb{R}_{+} ; \pi\right)$ is a dynamical semi-system, then $\left[\pi^{A}\right.$ is uniformly semi-stable $] \Leftrightarrow\left[\pi^{\bar{A}}\right.$ is uniformly semi-stable $]$, and $\left[\pi^{A}\right.$ is uniformly stable $] \Leftrightarrow\left[\pi^{\bar{A}}\right.$ is uniformly stable $]$.

R e m a r k 3.1. Stability of $\pi^{x}$ for every $x \in A$ is generally not sufficient for stability of $\pi^{\bar{A}}$. As an example consider a dynamical system with trajectories presented in Figure 1.

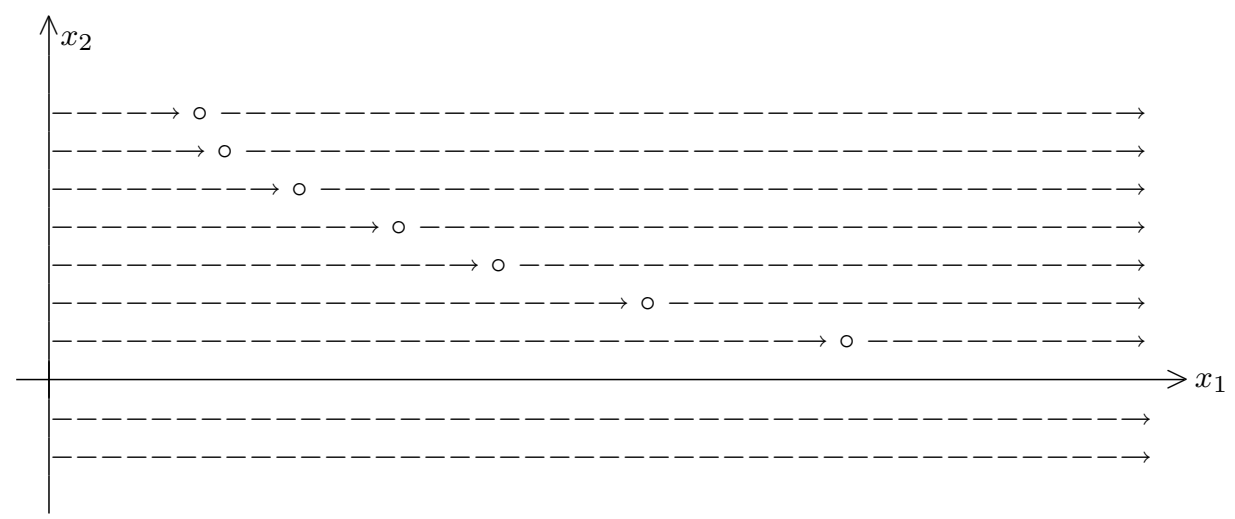

Fig. 1

The trajectory of $x=\left(x_{1}, x_{2}\right) \in X=\mathbb{R}^{2}$ with $x_{2}>0$ is the half-line parallel to the $x_{1}$-axis, having a one-element limit set (positive or negative, as clearly marked in the figure). Stationary points (small circles) lie on the graph of the function $x_{2}=\exp \left(-x_{1}\right)$. The trajectory of $\left(x_{1}, x_{2}\right)$ with $x_{2} \leq 0$ is the straight line parallel to the $x_{1}$-axis. We can define the motions of all points belonging to the set $A:=\left\{\left(0, x_{2}\right): x_{2}>0\right\}$ in such a way that every motion is positively stable. The point $(0,0)$ belongs to $\bar{A}$ but the motion $\pi^{(0,0)}$ cannot be positively stable (independently of the properties of the motions of points in $A$ ).

As another example consider a dynamical system discussed in [5], induced in $[-1,1] \times \mathbb{R}$ by the system of differential equations

$$
x^{\prime}=y\left(1-x^{2}\right), \quad y^{\prime}=-x .
$$


This system, considered here as a dynamical semi-system $\left(\bar{A}, \mathbb{R}_{+} ; \pi\right)$ with $A=(-1,1) \times \mathbb{R}$, has as trajectories closed orbits filling the set $A$ (surrounding the stationary point $(0,0))$, and two lines: $\{-1\} \times \mathbb{R}$ and $\{1\} \times \mathbb{R}$. For every $(x, y) \in A, \pi^{\pi((x, y))}$ is uniformly stable but $\pi^{A}$ is not uniformly stable and $\pi^{A}$ is not stable. In particular, $\pi^{(1,0)}$ is not stable; even the trajectory $\pi((1,0))$ is not stable as a set.

Re mark 3.2. If $A$ is such that $\mathbf{B}:=\overline{B(A, r)}$ is compact for some $r>0$ and $\pi$ is continuous in $\mathbf{B} \times \mathbb{R}_{+}$, then uniform semi-stability of $\pi^{A}$ in any set $D$ containing $A$ is equivalent to uniform stability of $\pi^{A}$ in $D$.

Corollary 3.2. If $\left(X, \mathbb{R}_{+} ; \pi\right)$ is a dynamical semi-system and $A$ is such that $\overline{B(A, r)}$ is compact for some $r>0$, then all the four conditions in the assertion of Corollary 3.1 are equivalent. If we assume, moreover, that $A$ is compact, then (see Proposition 2.1) each of those conditions is equivalent to stability of $\pi^{A}$.

4. Uniform semi-stability (stability) of $\pi^{A}$ implies semi-stability (stability) of $\pi^{B}$ for certain subsets $B$ of $\bar{A}$

THEOREM 4.1. Let $A$ be a nonempty subset of $X$, and let $D$ be a subset of $C(A, *)$ such that for every $x \in D$ there is $\beta>0$ such that

(4.1) $\quad \forall_{y \in B(x, \beta) \cap(A \cup D)} \exists_{s \geq 0} \forall_{t \geq s}\left(\left.\pi_{t}\right|_{B(x, \beta) \cap(A \cup D)}\right.$ is continuous at $\left.y\right)$.

If $\pi^{A}$ is uniformly stable then $\pi^{G}$, where $G:=A \cup D$, is semi-stable.

Proof. Fix $x \in D$. Suppose that $\pi^{x}$ is not semi-stable. So there are $\varepsilon>0$ and sequences $\left\{y_{n}\right\}$ of elements of $A \cup D$ and $\left\{t_{n}\right\}, t_{n}>0$, such that

$$
\begin{array}{cc}
y_{n} \rightarrow x & \text { as } n \rightarrow \infty, \\
t_{n} \rightarrow \infty & \text { as } n \rightarrow \infty
\end{array}
$$

$$
\varrho\left(\pi\left(t_{n}, x\right), \pi\left(t_{n}, y_{n}\right)\right)>\varepsilon \quad \text { for every } n .
$$

Since $\pi^{A}$ is uniformly stable, there are $\delta>0$ and $s \geq 0$ such that

$$
\{y, w \in A, \varrho(y, w)<\delta\} \Rightarrow\{\varrho(\pi(t, y), \pi(t, w))<\varepsilon / 4 \text { for } t \geq s\} .
$$

Let now $s_{1} \geq 0$ be such that for $t \geq s_{1}$ the mapping $\pi_{t}$ is continuous (as the mapping from $A \cup\{x\}$ into $X$; compare Section 1) at the point $x$. For every fixed $n$ such that $t_{n} \geq s_{1}$ we may find $\delta_{n}>0$ such that

$$
\left\{y \in A, \varrho(x, y)<\delta_{n}\right\} \Rightarrow \varrho\left(\pi\left(t_{n}, x\right), \pi\left(t_{n}, y\right)\right)<\varepsilon / 4 .
$$

Without loss of generality we may require that

$$
\delta_{n} \rightarrow 0 \quad \text { as } n \rightarrow \infty \text {. }
$$


For every fixed $n$ there is $k(n)$ such that $\varrho\left(x, y_{m}\right)<\delta_{n}$ for $m \geq k(n)$ (see (4.2)) and so (by (4.6))

$$
\varrho\left(\pi\left(t_{n}, x\right), \pi\left(t_{n}, y_{m}\right)\right)<\varepsilon / 4 \quad \text { for } m \geq k(n) .
$$

In particular, we have

$$
\varrho\left(\pi\left(t_{n}, x\right), \pi\left(t_{n}, y_{k(n)}\right)\right)<\varepsilon / 4 \quad \text { for } n \geq n^{*},
$$

where $n^{*}$ is chosen in such a way that $t_{n} \geq s_{1}$ for $n \geq n^{*}$. We may clearly assume that $k(n) \geq n$ (and so $k(n) \rightarrow \infty$ as $n \rightarrow \infty$ ).

Let now $\beta$ be such that the condition (4.1) holds true. If $n$ is large enough - for instance if $n \geq m^{*}$ - then

$$
\varrho\left(x, y_{k(n)}\right)<\beta \quad \text { and } \quad \varrho\left(x, y_{n}\right)<\beta
$$

and so, the $t_{n}$-translation restricted to the set $B(x, \beta) \cap(A \cup D)$ is continuous at $y_{k(n)}$ and at $y_{n}$ if $n \geq \max \left(n^{*}, m^{*}\right)$. Thus for every $n \geq \max \left(n^{*}, m^{*}\right)$ we can find $\eta_{n} \in(0, \beta)$ such that

(4.10) $\quad\left\{w \in A, \varrho\left(w, y_{k(n)}\right)<\eta_{n}\right\} \Rightarrow \varrho\left(\pi\left(t_{n}, w\right), \pi\left(t_{n}, y_{k(n)}\right)\right)<\varepsilon / 4$

as well as

$$
\left\{z \in A, \varrho\left(z, y_{n}\right)<\eta_{n}\right\} \Rightarrow \varrho\left(\pi\left(t_{n}, z\right), \pi\left(t_{n}, y_{n}\right)\right)<\varepsilon / 4 .
$$

We may require that $\eta_{n} \rightarrow 0$ as $n \rightarrow \infty$, and hence that

$$
\eta_{n}<\delta / 3 \text { for every } n \text {. }
$$

Since $y_{k} \in \bar{A}$ for every $k$, for every given $n$ we can find $w_{n}$ and $z_{n}$ in $A$ such that

$$
\varrho\left(w_{n}, y_{k(n)}\right)<\eta_{n} \quad \text { and } \quad \varrho\left(z_{n}, y_{n}\right)<\eta_{n} .
$$

Observe that (4.2) clearly gives $\varrho\left(y_{n}, y_{k(n)}\right) \rightarrow 0$ as $n \rightarrow \infty$, and so, for $n$ sufficiently large, say for $n \geq p^{*}$, we have

$$
\varrho\left(y_{n}, y_{k(n)}\right)<\delta / 3 \text {. }
$$

From (4.12)-(4.14) it follows that for $n \geq \max \left(n^{*}, m^{*}, p^{*}\right)$,

$$
\varrho\left(z_{n}, w_{n}\right)<\delta .
$$

This implies (by (4.5)) that

$$
\varrho\left(\pi\left(t_{n}, z_{n}\right), \pi\left(t_{n}, w_{n}\right)\right)<\varepsilon / 4
$$

for $n \geq \max \left(k^{*}, m^{*}, n^{*}, p^{*}\right)$, where $k^{*}$ is chosen in such a way that $t_{n} \geq s$ (see (4.5)) for $n \geq k^{*}$

On the other hand, (4.10), (4.11) and (4.13) imply that

$$
\varrho\left(\pi\left(t_{n}, w_{n}\right), \pi\left(t_{n}, y_{k(n)}\right)\right)<\varepsilon / 4
$$

and

$$
\varrho\left(\pi\left(t_{n}, z_{n}\right), \pi\left(t_{n}, y_{n}\right)\right)<\varepsilon / 4
$$


for $n \geq \max \left(n^{*}, m^{*}\right)$ (and so in particular, for $n \geq \max \left(k^{*}, m^{*}, n^{*}, p^{*}\right)$ ).

For $n \geq \max \left(k^{*}, m^{*}, n^{*}, p^{*}\right)$ we get

$$
\begin{aligned}
\varrho\left(\pi\left(t_{n}, x\right), \pi\left(t_{n}, y_{n}\right)\right) \leq & \varrho\left(\pi\left(t_{n}, x\right), \pi\left(t_{n}, y_{k(n)}\right)\right) \\
& +\varrho\left(\pi\left(t_{n}, y_{k(n)}\right), \pi\left(t_{n}, w_{n}\right)\right) \\
& +\varrho\left(\pi\left(t_{n}, w_{n}\right), \pi\left(t_{n}, z_{n}\right)\right) \\
& +\varrho\left(\pi\left(t_{n}, z_{n}\right), \pi\left(t_{n}, y_{n}\right)\right) .
\end{aligned}
$$

The consecutive members of the right hand side of (4.20) are each less than $\varepsilon / 4$ by (4.8), (4.18), (4.17) and (4.19), respectively. Thus the left hand side of (4.20) is less than $\varepsilon$, contrary to (4.4).

5. Some uniform stability conditions imply that prolongational limit sets are equal to corresponding generalized prolongational limit sets. Let $\left(X, \mathbb{R}_{+} ; \pi\right)$ be a pseudo-dynamical semi-system. It is well known (see for instance [1] or [3]) that if a motion $\pi^{x}$ is semi-stable, then the limit set and prolongational limit set of $x$ are equal. This extends in some sense to the properties of $\widehat{J}(\pi(x))$ under suitable uniform stability assumptions.

Theorem 5.1. Let $x \in X$ be fixed. If $\pi^{\pi(x)}$ is uniformly semi-stable in $\mathbf{B}:=B(\pi(x), \alpha)$ with some $\alpha>0$, then

$$
\widehat{J}(\pi(x))=J(x) .
$$

Proof. It is enough to prove that

$$
\widehat{J}(\pi(x)) \subseteq J(x)
$$

since the inverse inclusion is obvious. Let $y \in \widehat{J}(\pi(x))$. We have

$$
y=\lim \pi\left(t_{n}, x_{n}\right)
$$

where $t_{n} \rightarrow \infty, x_{n} \in X$ and

$$
\varrho\left(x_{n}, \pi(x)\right) \rightarrow 0 \quad \text { as } n \rightarrow \infty .
$$

This means that there are $s_{n} \geq 0$ such that

$$
\varrho\left(x_{n}, \pi\left(s_{n}, x\right)\right) \rightarrow 0 \quad \text { as } n \rightarrow \infty .
$$

Fix $\varepsilon \in(0, \alpha)$. Since $\pi^{\pi(x)}$ is uniformly semi-stable in $\mathbf{B}$, there are $\delta>0$ and $s \geq 0$ such that

(5.6) $\left\{z \in B, \varrho\left(z, \pi\left(s_{n}, x\right)\right)<\delta\right\} \Rightarrow\left\{\varrho\left(\pi(t, z), \pi\left(t+s_{n}, x\right)\right)<\varepsilon\right.$ for $\left.t \geq s\right\}$.

From (5.5) it follows that there is $n^{*}$ such that

$$
\varrho\left(x_{n}, \pi\left(s_{n}, x\right)\right)<\varepsilon \quad \text { for } n \geq n^{*} .
$$

We may of course require $n^{*}$ to be so large that

$$
t_{n} \geq s \quad \text { for } n \geq n^{*} .
$$


The conditions (5.6)-(5.8) imply

$$
\varrho\left(\pi\left(t_{n}, x_{n}\right), \pi\left(t_{n}+s_{n}, x\right)\right)<\varepsilon \quad \text { for } n \geq n^{*} .
$$

Thus we have proved that

$$
\varrho\left(\pi\left(t_{n}, x_{n}\right), \pi\left(t_{n}+s_{n}, x\right)\right) \rightarrow 0 \quad \text { as } n \rightarrow \infty .
$$

This implies (by (5.3)) that

$$
\pi\left(t_{n}+s_{n}, x\right) \rightarrow 0 \quad \text { as } n \rightarrow \infty .
$$

It is clear that $t_{n}+s_{n} \rightarrow \infty$ as $n \rightarrow \infty$, and so (5.10) means that $y \in J(x)$. The inclusion (5.2) is thus proved.

Corollary 5.1. Let $(X, \mathbb{R} ; \pi)$ be a pseudo-dynamical system. If $\pi^{\pi_{+}(x)}$ is uniformly positively semi-stable in $B\left(\pi_{+}(x), \alpha\right)$ (resp. $\pi^{\pi_{-}(x)}$ is uniformly negatively semi-stable in $B\left(\pi_{-}(x), \alpha\right)$ ) with some $\alpha>0$, then

$$
\widehat{J}^{+}\left(\pi_{+}(x)\right)=J^{+}(x) \quad\left(\text { resp. } \widehat{J}^{-}\left(\pi_{-}(x)\right)=J^{-}(x)\right) .
$$

Rem ark 5.1. Theorem 5.1 together with the well known result mentioned at the beginning of the present section: $\left[\pi^{x}\right.$ is semi-stable $] \Rightarrow[\Lambda(x)=$ $J(x)$ ], gives the following

COROLlary 5.2. If the assumptions of Theorem 5.1 are satisfied, then

$$
\widehat{J}(\pi(x))=J(x)=\Lambda(x) .
$$

R e m a r k 5.2. Uniform semi-stability of $\pi^{\pi(x)}$ in some set of the type $B(\pi(x), \alpha)$, assumed in Theorem 5.1 , cannot be replaced by uniform semistability (or even stability) of $\pi^{\pi(x)}$ in some open set containing $\pi(x)$.

Of course stability of $\pi^{\pi(x)}$ in $X$ is also not sufficient for the assertion of Theorem 5.1. As an example, consider a dynamical system on $X=\mathbb{R}^{2}$ having trajectories parallel to the $x_{1}$-axis (see Fig. 2).

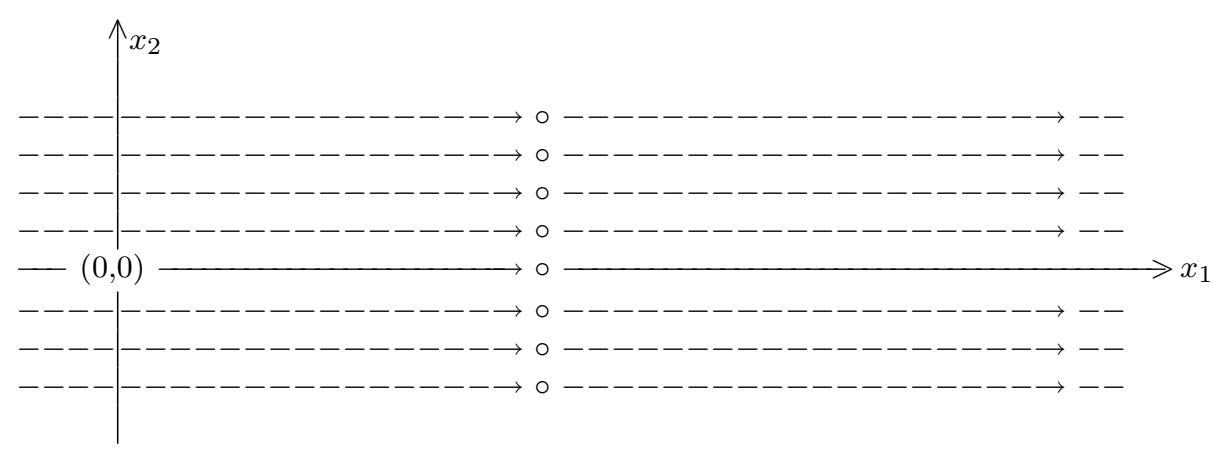

Fig. 2

Here the positive semi-trajectory of $x=(0,0)$ is $\left\{\left(x_{1}, 0\right): 0 \leq x_{1}<1\right\}$. It is easy to see that $\pi^{\pi(x)}$ is uniformly positively stable in the half plane 
$x_{1}<1$, but not in any set of the form $B(\pi(x), \alpha)$. It is also clear that $\pi^{\pi(x)}$ is positively stable in $X$. For $x=(0,0)$ we have

$$
\Lambda_{+}(x)=J^{+}(x)=\{(1,0)\} \neq \widehat{J}^{+}\left(\pi_{+}(x)\right)=\left\{\left(x_{1}, 0\right): x_{1} \geq 1\right\} .
$$

6. Uniform regularity of the mappings $x \longmapsto \Lambda(x), x \longmapsto J(x)$ and $x \longmapsto \widehat{J}(\pi(x))$. It is known from the theory of pseudo-dynamical systems (see for instance [2]) that positive semi-stability of $\pi^{x}$ implies upper semicontinuity of the mapping $y \longmapsto \Lambda_{+}(y)$ at $x$. Below we prove some results concerning uniform upper semi-continuity of the above mapping, as well as the mappings associating with $x$ various prolongational limit sets, under certain uniform stability assumptions, together with conditions of $\operatorname{Comp}[\alpha]$ type (see Section 1).

THEOREM 6.1. Let $\left(X, \mathbb{R}_{+} ; \pi\right)$ be a pseudo-dynamical semi-system and let $\mathcal{P}(X)$. If $\pi^{A}$ is uniformly semi-stable, then for every $\alpha>0$ such that $\mathcal{L}(A ; \alpha) \neq \emptyset$ the mapping

$$
\mathcal{L}^{+}(A ; \alpha) \ni x \longmapsto \Lambda(x) \in \mathcal{P}(X)
$$

is uniformly upper semi-continuous in $\mathcal{L}^{+}(A ; \alpha)$.

Proof. Let $\alpha>0$ be such that $\mathcal{L}(A ; \alpha) \neq \emptyset$ and let $\varepsilon>0$. Put $\eta:=$ $\min (\alpha, \varepsilon / 2)$ and take $\delta>0$ and $s \geq 0$ such that

(6.2) $\{x \in A, y \in A, \varrho(x, y)<\delta\} \Rightarrow\{\varrho(\pi(t, x), \pi(t, y))<\eta / 2$ for $t \geq s\}$.

Fix $x, y \in \mathcal{L}(A ; \alpha)$. Take $z \in \Lambda(x)$. There are $t_{n}>0$ such that

$$
t_{n} \rightarrow \infty \text { and } \pi\left(t_{n}, x\right) \rightarrow z \quad \text { as } n \rightarrow \infty .
$$

For $n$ large enough we have $t_{n} \geq s$ and so

$$
\varrho\left(\pi\left(t_{n}, x\right), \pi\left(t_{n}, y\right)\right)<\eta / 2
$$

for $n$ sufficiently large, say for $n \geq n^{*}$. By (6.2) we have for sufficiently large $n$, say for $n \geq m^{*}$,

$$
\varrho\left(\pi\left(t_{n}, x\right), z\right)<\eta / 2
$$

and then for $n \geq \max \left(n^{*}, m^{*}\right)$,

$$
\varrho\left(\pi\left(t_{n}, y\right), z\right)<\eta
$$

Thus for $n \geq \max \left(n^{*}, m^{*}\right)$,

$$
\pi\left(t_{n}, y\right) \in \overline{B(z, \eta)} \subseteq \overline{B(z, \alpha)}
$$

and by the compactness of $\overline{B(z, \alpha)}$ ( since $\Lambda(x) \in \operatorname{Comp}[\alpha]$ ) we may assume that the sequence $\left\{\pi\left(t_{n}, y\right)\right\}$ is convergent to some $w \in \overline{B(z, \alpha)}$. Clearly, $z \in \overline{B(w, \alpha)}$. On the other hand, $w \in \Lambda(y)$ and so

$$
z \in \overline{B(\Lambda(y), \alpha)} \subseteq B(\Lambda(y), \varepsilon) .
$$


We have proved that $\Lambda(x) \subseteq B(\Lambda(y), \varepsilon)$. Similarly we prove that $\Lambda(y) \subseteq$ $B(\Lambda(x), \varepsilon)$.

R e mark 6.1. It is easy to see that the proof of Theorem 6.1 also works under the slightly weaker assumption that $\pi^{\mathcal{L}(A ; \alpha)}$ is uniformly stable.

If $\pi^{x}$ is semi-stable, then (as already mentioned in Section 5) $J(x)=$ $\Lambda(x)$. So if $\pi^{A}$ is semi-stable in some open set $D$ containing $A$, then the sets (1.14) and (1.15) are equal. Hence, from Theorem 6.1 we obtain the following

COROllary 6.1. If $\pi^{A}$ is uniformly semi-stable and $\pi^{A}$ is semi-stable in some open set $D$ containing $A$, then for every $\alpha>0$ such that $\mathcal{J}(A ; \alpha) \neq \emptyset$, the mapping

$$
\mathcal{J}(A ; \alpha) \ni x \longmapsto J(x) \in \mathcal{P}(X)
$$

is uniformly upper semi-continuous in $\mathcal{J}(A ; \alpha)$.

Using Theorem 5.1 we get immediately from Theorem 6.1 the next corollary concerning regularity of the mapping associating with $x$ the set $\widehat{J}(\pi(x))$ :

COROLlary 6.2. If $\pi^{A}$ is uniformly positively semi-stable in a set $B(A, \beta)$ for some $\beta>0$, then for every $\alpha>0$ such that $\widehat{\mathcal{J}}(A ; \alpha) \neq \emptyset$ the mapping

$$
\widehat{\mathcal{J}}(A ; \alpha) \ni x \longmapsto \widehat{J}(\pi(x)) \in \mathcal{P}(X)
$$

is uniformly upper semi-continuous in $\widehat{\mathcal{J}}(A ; \alpha)$.

7. Lyapunov functions. We present below some results, similar to the classical ones, concerning connections between uniform stability properties of motions and existence of some functions of Lyapunov type.

We first introduce some terminology.

Let $\left(X, \mathbb{R}_{+} ; \pi\right)$ be a pseudo-dynamical semi-system. Assume that $A, D$, $E$ are subsets of $X$ such that $A \neq \emptyset, A \subseteq D \subseteq E$ and $E$ is invariant, which means that

$$
x \in E \Rightarrow \pi(x) \subseteq E .
$$

Let $W$ be a function from $\mathbb{R}_{+} \times E \times E$ into $\mathbb{R}_{+}$such that

(i) $\forall_{\varepsilon>0} \exists_{\delta>0}(\{x \in A, y \in D, \varrho(x, y)<\delta\} \Rightarrow W(0, x, y)<\varepsilon)$,

(ii) $\forall_{\eta>0} \exists_{\gamma>0}(\{x \in A, y \in D, \varrho(x, y) \geq \eta\} \Rightarrow\{W(t, x, y) \geq \gamma$ for $t \geq 0\}$ ),

(iii) $\{x \in A, y \in D, t \geq 0\} \Rightarrow W(t, \pi(t, x), \pi(t, y)) \leq W(0, x, y)$.

Such a function will be called a time-dependent Lyapunov function for the pair $(A, D)$ (in the semi-system $\left(X, \mathbb{R}_{+} ; \pi\right)$ ), or briefly a $\tau$-Lyapunov function for $(A, D)$. 
THEOREM 7.1. If there exists a $\tau$-Lyapunov function $W: \mathbb{R}_{+} \times E \times E \rightarrow$ $\mathbb{R}_{+}$(where $E$ is some invariant subset of $X$ such that $A \subseteq D \subseteq E$ ) then $\pi^{A}$ is uniformly stable in $D$.

Proof. Let $\varepsilon>0$. Take $\gamma>0$ such that (see (ii))

$$
\{x \in A, y \in D, \varrho(x, y) \geq \varepsilon\} \Rightarrow\{W(t, x, y) \geq \gamma \text { for } t \geq 0\} .
$$

We have to show that for every $\delta>0$,

(7.3) $\{x \in A, y \in D, \varrho(x, y)<\delta\} \Rightarrow\{\varrho(\pi(t, x), \pi(t, y))<\varepsilon$ for $t \geq 0\}$.

Assume the contrary. Then there are $\left\{x_{n}\right\},\left\{y_{n}\right\}$ and $\left\{t_{n}\right\}$ such that for every $n$,

$$
\varrho\left(x_{n}, y_{n}\right)<1 / n
$$

but

$$
\varrho\left(\pi\left(t_{n}, x_{n}\right), \pi\left(t_{n}, y_{n}\right)\right) \geq \varepsilon .
$$

Take now $\beta>0$ such that (see (i))

$$
\{x \in A, y \in D, \varrho(x, y)<\beta\} \Rightarrow W(0, x, y)<\gamma / 2 .
$$

We have $1 / n<\beta$ for $n$ sufficiently large and so, for such $n$,

$$
\varrho\left(x_{n}, y_{n}\right)<\beta \text {, }
$$

which implies, because of (7.6), the inequality

$$
W\left(0, x_{n}, y_{n}\right)<\gamma / 2
$$

for $n$ large enough.

The condition (7.5) implies for every fixed $n$ the inequality

$$
W\left(t_{n}, \pi\left(t_{n}, x_{n}\right), \pi\left(t_{n}, y_{n}\right)\right) \geq \gamma .
$$

On the other hand, we have (by (iii))

$$
W\left(t_{n}, \pi\left(t_{n}, x_{n}\right), \pi\left(t_{n}, y_{n}\right)\right) \leq W\left(0, x_{n}, y_{n}\right) .
$$

So for $n$ large enough we have (see (7.7))

$$
W\left(t_{n}, \pi\left(t_{n}, x_{n}\right), \pi\left(t_{n}, y_{n}\right)\right)<\gamma / 2,
$$

which contradicts (7.8).

Theorem 7.2. Assume that $A, D \in \mathcal{P}(X)$ are such that $A \subseteq D$. If $\pi^{A}$ is uniformly stable in $D$ then there exists an invariant subset $E$ of $X$ containing $D$ and there exists a function

$$
V: E \times E \rightarrow \mathbb{R}_{+}
$$

such that

(j) $\forall_{\varepsilon>0} \exists_{\delta>0}(\{x \in A, y \in D, \varrho(x, y)<\delta\} \Rightarrow V(x, y)<\varepsilon)$,

(jj) $\forall_{\eta>0} \exists_{\gamma>0}(\{x \in A, y \in D, \varrho(x, y) \geq \eta\} \Rightarrow V(x, y) \geq \gamma)$, 
(jjj) $\{x, y \in D, t \geq 0\} \Rightarrow V(\pi(t, x), \pi(t, y)) \leq V(x, y)$.

Proof. Assume that $\pi^{A}$ is uniformly stable in $D$. Put $E:=\pi(D)$, where

$$
\pi(D):=\{\pi(t, x): t \geq 0, x \in D\} .
$$

The set $E$ is clearly invariant. For $x, y \in E$ define

$$
V_{0}(x, y):=\sup \{\varrho(\pi(t, x), \pi(t, y)): t \geq 0\} .
$$

To exclude the case $V_{0}(x, y)=\infty$, define for $(x, y) \in E \times E$,

$$
V(x, y):=\min \left\{1, V_{0}(x, y)\right\} .
$$

We now show that $V$ satisfies the conditions $(\mathrm{j})-(\mathrm{jjj})$.

Let $\varepsilon>0$. Take any $\varepsilon^{\prime} \in(0, \varepsilon)$. Since $\pi^{A}$ is uniformly stable, there is $\delta>0$ such that

$$
\{x \in A, y \in D, \varrho(x, y)<\delta\} \Rightarrow\left\{\varrho(\pi(t, x), \pi(t, y))<\varepsilon^{\prime} \text { for } t \geq 0\right\} .
$$

This shows that if $\varrho(x, y)<\delta$ then $V_{0}(x, y)<\varepsilon$ and so of course also $V(x, y)<\varepsilon$. The condition $(\mathrm{j})$ is satisfied.

Let now $\eta>0$. Assume first that $\eta \leq 1$. Suppose that $x \in A$ and $y \in D$ are such that $\varrho(x, y) \geq \eta$. Since $x=\pi(0, x), y=\pi(0, y)$, we have clearly $V_{0}(x, y) \geq \eta$. This implies $V(x, y) \geq \eta$ as $\eta \leq 1$. Thus, for $\eta \leq 1$, it is enough to put $\gamma=\eta$ in order to get the implication required in (jj).

Assume now that $\eta>1$. Applying the implication in (jj) with $\eta=1$ and $\gamma=\eta$ we observe that

$$
\begin{aligned}
\{x \in A, y \in D, \varrho(x, y) \geq \eta\} & \Rightarrow\{x \in A, y \in D, \varrho(x, y) \geq 1\} \\
& \Rightarrow V(x, y) \geq 1,
\end{aligned}
$$

proving the required implication with $\gamma:=\min \{1, \eta\}$ for any fixed $\eta>0$.

In order to prove (jjj), fix $x, y \in D$ and $t \geq 0$. We have

$$
V_{0}(\pi(t, x), \pi(t, y)) \leq V_{0}(x, y),
$$

since the left hand side is clearly the supremum of the same quantity as on the right hand side, but over a smaller set. The inequality for $V$ follows trivially.

Remark 7.1. It seems natural to call any function (7.10) satisfying (j)-(jjj) a (time-independent) Lyapunov function for the pair $(A, D)$ in the semi-system $\left(X, \mathbb{R}_{+} ; \pi\right)$ or briefly, a Lyapunov function for $(A, D)$.

R e m a r k 7.2. The assertion of Theorem 7.2 does not give any particular information on properties of the set $E$, except its invariance and the fact that it contains $D$. In several specific questions (including applications to dynamical systems induced by ordinary differential equations) it might be important to know that the set $E$ has nonempty interior and that $D \subseteq$ int $E$. This excludes possible triviality, which can be produced if, for instance, 
$A=D=E$. It is, however, not difficult to modify the proof of Theorem 7.2 in such a way that int $E \supseteq D$. Indeed, consider $D^{\prime}:=B(D, \alpha)$ with some fixed $\alpha>0$, and $E:=\pi\left(D^{\prime}\right)$.

As a simple, but important, corollary of the above Theorems 7.1 and 7.2 (together with Remark 7.2) we get the following

Theorem 7.3. Let $A, D \in \mathcal{P}(X)$ be such that $A \subseteq D$. The following conditions are equivalent:

(I) There is an invariant set $E$ containing $D$ and there exists a $\tau$-Lyapunov function $W: \mathbb{R}_{+} \times E \times E \rightarrow \mathbb{R}_{+}$for $(A, D)$.

(II) There is an invariant set $E$ such that $D \subseteq \operatorname{int} E$ and there exists a Lyapunov function $V: E \times E \rightarrow \mathbb{R}_{+}$for $(A, D)$.

(III) $\pi^{A}$ is uniformly stable in $D$.

Proof. We need only prove the implication (II) $\Rightarrow(\mathrm{I})$, which is trivial, because having $V$ from (II) we put simply $W(t, x, y):=V(x, y)$ for $x, y \in E$ and $t \geq 0$, getting $W$ which satisfies the conditions (i)-(iii).

Remark 7.3. In pseudo-dynamical systems we consider positively and negatively invariant sets and suitable Lyapunov functions corresponding to positively and negatively stable motions.

8. Extensions of asymptotic equivalence concepts and uniform stability properties. We shall deal with relations between some asymptotic equivalence conditions for pairs of pseudo-dynamical semi-systems and their uniform stability properties.

Denote by $\Phi$ the family of all functions $\phi: \mathbb{R}_{+} \rightarrow \mathbb{R}_{+}$such that $\lim _{\varepsilon \rightarrow 0} \phi(\varepsilon)=0$ and $\phi$ is nondecreasing in $[0, \beta)$ with some positive $\beta$. Set $\Phi^{0}:=\{\phi \in \Phi: \phi(\varepsilon)>0$ for $\varepsilon>0\}$.

Let $\left(X, \mathbb{R}_{+} ; \pi\right)$ and $\left(X, \mathbb{R}_{+} ; \sigma\right)$ be two pseudo-dynamical semi-systems, fixed throughout this section. Let $x, y \in X$ and $\phi \in \Phi$. We say that $\pi^{x}$ approaches eventually $\sigma^{y}$ with error $\phi$ (briefly: $\pi^{x} \mathrm{M}(\phi) \mathrm{AE} \sigma^{y}$ ) if for every $\varepsilon>0$ there are $\delta>0$ and $T \geq 0$ such that

$$
\pi_{t}(B(x, \delta)) \subseteq B\left(\sigma_{t}(B(y, \varepsilon)), \phi(\varepsilon)\right) \quad \text { for } t \geq T,
$$

and that $\pi^{x}$ weakly approaches eventually $\sigma^{y}$ with error $\phi$ (briefly: $\left.\pi^{x} \mathrm{wM}(\phi) \mathrm{AE} \sigma^{y}\right)$ if for every $\varepsilon>0$ there is $T \geq 0$ such that

$$
\pi(t, x) \in B\left(\sigma_{t}(B(y, \varepsilon)), \phi(\varepsilon)\right) \quad \text { for } t \geq T .
$$

Remark 8.0. We adopt here the following convention: for $C \in \mathcal{P}(X)$ we put $B(C, 0):=C$ and we do not exclude $\phi$ vanishing on some interval.

Let now $C \in \mathcal{P}(X)$ be given. We say that $\pi^{x}$ approaches eventually $\sigma^{y}$ in $C$ with error $\phi$ (briefly: $\pi^{x} \mathrm{M}(\phi) \mathrm{AE}_{C} \sigma^{y}$ ) if for every $\varepsilon>0$ there are 
$\delta>0$ and $T \geq 0$ such that

$$
\pi_{t}(B(x, \delta) \cap C) \subseteq B\left(\sigma_{t}(B(y, \varepsilon)), \phi(\varepsilon)\right) \quad \text { for } t \geq T .
$$

Remark 8.1. If $C$ is open, then

$$
\pi^{x} \mathrm{M}(\phi) \mathrm{AE}_{C} \sigma^{y} \Leftrightarrow \pi^{x} \mathrm{M}(\phi) \mathrm{AE} \sigma^{y},
$$

and in particular,

$$
\pi^{x} \mathrm{M}(\phi) \mathrm{AE}_{X} \sigma^{y} \Leftrightarrow \pi^{x} \mathrm{M}(\phi) \mathrm{AE} \sigma^{y} .
$$

Remark 8.2. Let $\mathbf{0}$ be the zero function. Then $\mathbf{0} \in \Phi$. The condition $\pi^{x} \mathrm{M}(\mathbf{0}) \mathrm{AE} \sigma^{y}$ means that for every $\varepsilon>0$ there are $\delta>0$ and $T \geq 0$ such that (compare Remark 8.0)

$$
\pi_{t}(B(x, \delta)) \subseteq \sigma_{t}(B(y, \varepsilon)) \quad \text { for every } t \geq T .
$$

This condition is considered in [4] (see also [3]) and written as $\pi^{x} \mathrm{AE} \sigma^{y}$ $\left(\pi^{x}\right.$ approaches eventually $\left.\sigma^{y}\right)$. It is in fact a modification of the condition introduced by T. Ważewski in [7] and called asymptotic coincidence of trajectories of ordinary differential equations (see also [6] for its extensions and [4] for certain details and a further discussion).

Remark 8.3. It is obvious that for every $\phi \in \Phi$ and $C \in \mathcal{P}(X)$,

$$
\pi^{x} \mathrm{M}(\phi) \mathrm{AE}_{C} \sigma^{y} \Rightarrow \pi^{x}{ }_{\mathrm{wM}}(\phi) \mathrm{AE}_{C} \sigma^{y},
$$

but not conversely in general. If, however, $\phi \in \Phi^{0}$ and $\pi^{x}$ is semi-stable, then the implication inverse to (8.6) is true and so both conditions in (8.6) are equivalent.

Indeed, if $\pi^{x} \operatorname{wM}(\phi) \mathrm{AE}_{C} \sigma^{y}$ (with $\phi \in \Phi^{0}$ ) and $\varepsilon>0$, then we can find $T \geq 0$ such that (8.2) holds with $\varepsilon$ replaced by $\varepsilon^{\prime} \in(0, \varepsilon)$ such that $\phi\left(\varepsilon^{\prime}\right)<\phi(\varepsilon) / 2$, and then we find $s \geq 0$ and $\delta>0$ such that

$$
\{z \in C, \varrho(x, z)<\delta\} \Rightarrow\left\{\varrho(\pi(t, x), \pi(t, z))<\phi\left(\varepsilon^{\prime}\right) \text { for } t \geq s\right\} .
$$

For $t \geq \max (s, T)$ this gives

$$
\pi_{t}(B(x, \delta) \cap C) \subseteq B\left(\sigma_{t}\left(B\left(y, \varepsilon^{\prime}\right)\right), \phi(\varepsilon)\right) \subseteq B\left(\sigma_{t}(B(y, \varepsilon)), \phi(\varepsilon)\right) .
$$

Corollary 8.1. If $\phi \in \Phi^{0}$ and $\pi^{x}$ is semi-stable, then

$$
\pi^{x} \mathrm{M}(\phi) \mathrm{AE} \sigma^{y} \Leftrightarrow \pi^{x} \mathrm{wM}(\phi) \mathrm{AE} \sigma^{y} .
$$

It is clear that we are not able to use the above arguments if $\phi$ does not belong to $\Phi^{0}$; in particular, for $\phi$ vanishing identically the condition (8.2) is essentially weaker than (8.1), and even stability of the motion $\pi^{x}$ gives - in general - no possibilities of proving the implication inverse to (8.6). This observation shows that the concept of "approaching with some error" seems to be a reasonable generalization of the condition denoted in [3] and [4] by " $\pi^{x} \mathrm{AE} \sigma^{y "}$. 
If $\phi$ belongs to $\Phi$ (and not necessarily to $\Phi^{0}$ ), then we can prove a weaker version of the assertion of Corollary 8.1:

Re mark 8.4. If $\phi \in \Phi, \pi^{x}$ is semi-stable, $C \in \mathcal{P}(X)$ and $\psi \in \Phi^{0}$ is such that $\phi(\varepsilon) \leq \psi(\varepsilon)$ for every $\varepsilon$, then

$$
\pi^{x} \operatorname{wM}(\phi) \mathrm{AE}_{C} \sigma^{y} \Rightarrow \pi^{x} \mathrm{M}(\psi) \mathrm{AE}_{C} \sigma^{y} .
$$

Indeed, if $\lambda, \mu \in \Phi$ and $\lambda(\varepsilon) \leq \mu(\varepsilon)$ for every $\varepsilon$, then

$$
\pi^{x} \mathrm{wM}(\lambda) \mathrm{AE}_{C} \sigma^{y} \Rightarrow \pi^{x}{ }_{\mathrm{wM}}(\mu) \mathrm{AE}_{C} \sigma^{y} ;
$$

it is now enough to use Remark 8.3.

As a special case of $\psi$ we can consider for instance

$$
\psi(\varepsilon):=\max (a \varepsilon, \phi(\varepsilon)) \quad \text { for } \varepsilon \geq 0,
$$

where $a$ is a fixed positive number.

We say that $\pi^{x}$ and $\sigma^{y}$ are asymptotically equivalent with error $\phi$ (briefly: $\pi^{x}$ and $\sigma^{y}$ are $\left.\mathrm{M}_{\phi} \mathrm{AE}\right)$ if $\pi^{x} \mathrm{M}(\phi) \mathrm{AE} \sigma^{y}$ and $\sigma^{y} \mathrm{M}(\phi) \mathrm{AE} \pi^{x}$.

It is obvious that if $\pi^{x} \mathrm{M}\left(\phi_{1}\right) \mathrm{AE} \sigma^{y}$ and $\sigma^{y} \mathrm{M}\left(\phi_{2}\right) \mathrm{AE} \pi^{x}$ then $\pi^{x}$ and $\sigma^{y}$ are $\mathrm{M}_{\phi} \mathrm{AE}$, with $\phi(\varepsilon):=\max \left\{\phi_{1}(\varepsilon), \phi_{2}(\varepsilon)\right\}$; if $\phi_{1}, \phi_{2} \in \Phi^{0}$, then $\phi \in \Phi^{0}$.

We say that $\pi^{x}$ and $\sigma^{y}$ are weakly asymptotically equivalent with error $\phi$ (briefly: $\pi^{x}$ and $\sigma^{y}$ are $\mathrm{wM}_{\phi} \mathrm{AE}$ ) if $\pi^{x} \mathrm{wM}(\phi) \mathrm{AE} \sigma^{y}$ and $\sigma^{y} \mathrm{wM}(\phi) \mathrm{AE} \pi^{x}$.

Using Remark 8.3 we can state the following simple

Rem ark 8.5. If $\pi^{x}$ and $\sigma^{y}$ are semi-stable and $\phi \in \Phi^{0}$, then

$$
\left\{\pi^{x} \text { and } \sigma^{y} \text { are } \mathrm{wM}_{\phi} \mathrm{AE}\right\} \Leftrightarrow\left\{\pi^{x} \text { and } \sigma^{y} \text { are } \mathrm{wM}_{\phi} \mathrm{AE}\right\} \text {. }
$$

Let now $B, C \in \mathcal{P}(X)$ be such that $B \subseteq C$, let $\phi \in \Phi$ be given and let $f$ be a function from $C$ into $X$. We shall say that $\pi^{B}$ approaches eventually $\sigma^{f(B)}$ in $C$ with error $\phi$ (briefly: $\left.\pi^{B} \mathrm{M}(\phi) \mathrm{AE}_{C} \sigma^{f(B)}\right)$ if $\pi^{x} \mathrm{M}(\phi) \mathrm{AE} \sigma^{f(x)}$ for every $x \in B$. We say that $\pi^{B}$ uniformly approaches eventually $\sigma^{f(B)}$ in $C$ with error $\phi\left(\pi^{B} \mathrm{M}(\phi) \mathrm{UAE}_{C} \sigma^{f(B)}\right)$ if for every $\varepsilon>0$ there exist $\delta>0$ and $T \geq 0$ such that for every $x \in C$ the condition (8.3) is satisfied for $y=f(x)$. We say that $\pi^{B}$ approaches eventually $\sigma^{f(B)}$ with error $\phi\left(\pi^{B} \mathrm{M}(\phi) \mathrm{AE}\right.$ $\left.\sigma^{f(B)}\right)$ if $\pi^{x} \mathrm{M}(\phi) \mathrm{AE} \sigma^{f(x)}$ for every $x \in X$, and that $\pi^{B}$ uniformly approaches eventually $\sigma^{f(B)}$ with error $\phi$ (briefly: $\pi^{B} \mathrm{M}(\phi) \mathrm{UAE} \sigma^{f(B)}$ ) if $\pi^{B} \mathrm{M}(\phi) \mathrm{UAE}_{X} \sigma^{f(B)}$. We say that $\pi^{B}$ weakly uniformly approaches eventually $\sigma^{f(B)}$ in $C$ with error $\phi\left(\pi^{B} \mathrm{wM}(\phi) \mathrm{UAE} \sigma^{f(B)}\right)$ if for every $\varepsilon>0$ there exists $T \geq 0$ such that for each $x \in C$ the condition (8.2) holds with $y=f(x)$ being the center of the ball appearing in the right hand side member of the inclusion.

R e mark 8.6. Using arguments similar to those applied in the proof of the statement of Remark 8.3 , we can prove that if $\pi^{B}$ is uniformly semi- 
stable in some open set containing $B$ and $\phi \in \Phi^{0}$, then

$$
\pi^{B} \mathrm{wM}(\phi) \mathrm{UAE} \sigma^{f(B)} \Leftrightarrow \pi^{B} \mathrm{M}(\phi) \mathrm{UAE} \sigma^{f(B)} .
$$

We omit the details.

Let $B, C, D, G \in \mathcal{P}(X)$ be such that $B \subseteq C, D \subseteq G$, and let $f: C \rightarrow$ $G$ be a bijection such that $f(B)=D$. We say that $\left(\pi^{B}, C\right)$ and $\left(\sigma^{D}, G\right)$ are asymptotically $f$-equivalent with error $\phi$ (briefly: $\left(\pi^{B}, C\right)$ and $\left(\sigma^{D}, G\right)$ are $f-\mathrm{M}(\phi) \mathrm{AE})$ if $\pi^{B} \mathrm{M}(\phi) \mathrm{AE}_{C} \sigma^{D}$ and $\sigma^{D} \mathrm{M}(\phi) \mathrm{AE}_{G} \pi^{B}$. We say that $\left(\pi^{B}, C\right)$ and $\left(\sigma^{D}, G\right)$ are uniformly asymptotically $f$-equivalent with error $\phi$ (briefly: $\left(\pi^{B}, C\right)$ and $\left(\sigma^{D}, G\right)$ are $\left.f-\mathrm{M}(\phi) \mathrm{UAE}\right)$ if $\pi^{B} \mathrm{M}(\phi) \mathrm{UAE}_{C} \sigma^{D}$ and $\sigma^{D} \mathrm{M}(\phi) \mathrm{UAE}_{G} \pi^{B}$.

Theorem 8.1. Let $B, C \in \mathcal{P}(X)$ be such that $B \subseteq C$. Assume that $B$ is compact, $f: C \rightarrow X$ is continuous, $\phi \in \Phi^{0}$ and $\sigma^{f(B)}$ is uniformly semi-stable in $X$. Then

$$
\pi^{B} \mathrm{M}(\phi) \mathrm{UAE}_{C} \sigma^{f(B)} \Leftrightarrow \pi^{B} \mathrm{M}(\phi) \mathrm{AE}_{C} \sigma^{f(B)} .
$$

Proof. It is enough to prove the implication $\Leftarrow$. Assume that $\pi^{B} \mathrm{M}(\phi) \mathrm{AE}_{C} \sigma^{f(B)}$, but there exists $\varepsilon^{0}>0$ such that for every $\delta>0$ and for every $T \geq 0$ there are $x \in B$ and $t \geq T$ such that

$$
\pi_{t}(B(x, \delta) \cap C) \cap\left\{X \backslash B\left(\sigma_{t}\left(B\left(f(x), \varepsilon^{0}\right)\right), \phi\left(\varepsilon^{0}\right)\right)\right\} \neq \emptyset .
$$

Thus for every $n$ there are $x_{n} \in B$ and $t_{n} \geq n$ such that for some $y_{n} \in$ $B\left(x_{n}, 1 / n\right) \cap C$,

$$
\pi\left(t_{n}, y_{n}\right) \notin B\left(\sigma\left(t_{n} ; B\left(f\left(x_{n}\right), \varepsilon^{0}\right)\right), \phi\left(\varepsilon^{0}\right)\right)
$$

(notation defined at the end of Section 1).

Without loss of generality we may assume (by compactness of $B$ ) that $\left\{x_{n}\right\}$ is convergent to some $x^{0} \in B$. Of course $\left\{y_{n}\right\}$ also converges to $x^{0}$. Since $f$ is continuous, the sequences $\left\{f\left(x_{n}\right)\right\}$ and $\left\{f\left(y_{n}\right)\right\}$ converge to $f\left(x^{0}\right)$.

Let now $\varepsilon^{\prime} \in(0, \varepsilon)$ be such that $\phi\left(\varepsilon^{\prime}\right)<\phi\left(\varepsilon^{0}\right) / 8$ and let $\gamma \in\left(0, \varepsilon^{\prime}\right)$ and $T^{\prime} \geq 0$ be chosen in such a way that

$$
\begin{aligned}
\left\{z \in X, \varrho\left(f\left(x^{0}\right), z\right)<\gamma\right\} & \\
& \Rightarrow\left\{\varrho\left(\sigma\left(t, f\left(x^{0}\right)\right), \sigma(t, z)\right)<\phi\left(\varepsilon^{\prime}\right) \text { for } t \geq T^{\prime}\right\} .
\end{aligned}
$$

For $n$ sufficiently large, say for $n \geq n^{*}$, we have

$$
\varrho\left(f\left(x_{n}\right), f\left(x^{0}\right)\right)<\gamma \quad \text { and } \quad t_{n} \geq T^{\prime}
$$

and so, for such $n$, we have

$$
\varrho\left(\sigma\left(t_{n}, f\left(x^{0}\right)\right), \sigma\left(t_{n}, f\left(x_{n}\right)\right)\right)<\phi\left(\varepsilon^{\prime}\right) .
$$

For any $D \in \mathcal{P}(X)$ we put

$$
d[D]:=\sup \{\varrho(x, y): x, y \in D\} \quad(\text { the diameter of } D) .
$$


It is clear that for $n \geq n^{*}$ we have

$$
d\left[\sigma\left(t_{n} ; B\left(f\left(x^{0}\right), \gamma\right)\right)\right]<2 \phi\left(\varepsilon^{\prime}\right)
$$

and

$$
d\left[\sigma\left(t_{n} ; B\left(f\left(x_{n}\right), \gamma\right)\right)\right]<2 \phi\left(\varepsilon^{\prime}\right) .
$$

The conditions (8.12)-(8.14) give, for $n \geq n^{*}$,

$$
d\left[\sigma\left(t_{n} ; B\left(f\left(x_{n}\right), \gamma\right)\right) \cup \sigma\left(t_{n} ; B\left(f\left(x^{0}\right), \gamma\right)\right)\right]<5 \phi\left(\varepsilon^{\prime}\right) .
$$

We have assumed that $\pi^{x} \mathrm{M}(\phi) \mathrm{AE}_{C} \sigma^{f(x)}$ for every $x \in B$; in particular, there are $\delta^{0}>0$ and $T^{0} \geq 0$ such that

$$
\pi_{t}\left(B\left(x^{0}, \delta^{0}\right)\right) \subseteq B\left(\sigma\left(t ; B\left(f\left(x^{0}\right), \gamma\right)\right), \phi(\gamma)\right) \quad \text { for } t \geq T^{0} .
$$

If $n$ is large enough, say $n \geq m^{*}$, then $\varrho\left(x^{0}, y_{n}\right)<\delta^{0}$ and $t_{n} \geq T^{0}$ and so

$$
\pi\left(t_{n}, y_{n}\right) \in B\left(\sigma\left(t_{n} ; B\left(f\left(x^{0}\right), \gamma\right)\right), \phi(\gamma)\right) .
$$

Thus, for $n \geq m^{*}$,

$$
\pi\left(t_{n}, y_{n}\right) \in B\left(\sigma\left(t_{n} ; B\left(f\left(x^{0}\right), \gamma\right)\right), \phi(\gamma)\right) \cup \sigma\left(t_{n} ; B\left(f\left(x_{n}\right), \gamma\right)\right) .
$$

Since $\phi(\gamma) \leq \phi\left(\varepsilon^{\prime}\right)$ (recall that $\gamma<\varepsilon^{\prime}$ ), we have, by (8.15), for $n \geq$ $\max \left(n^{*}, m^{*}\right)$,

(8.19) $d\left[B\left(\sigma\left(t_{n} ; B\left(f\left(x_{n}\right), \phi(\gamma)\right) \cup B\left(\sigma\left(t_{n} ; B\left(f\left(x^{0}\right), \gamma\right)\right), \phi(\gamma)\right)\right]<7 \phi\left(\varepsilon^{\prime}\right)\right.\right.$.

Denote by $D_{n}$ the set in square brackets in (8.15). Observe that if $z \in$ $D_{n}$, then $z \in B\left(Q, 8 \phi\left(\varepsilon^{\prime}\right)\right)$ for each $n \geq \max \left(n^{*}, m^{*}\right)$ and each subset $Q$ of $D_{n}$. Thus, in particular, $\pi\left(t_{n}, y_{n}\right)$ belonging to $D_{n}$ (see (8.18)) must for $n \geq \max \left(n^{*}, m^{*}\right)$, also belong to the set

$$
B\left(\sigma\left(t_{n} ; B\left(f\left(x_{n}\right), \gamma\right)\right), 8 \phi(\gamma)\right) .
$$

The set (8.20) is, however, contained in

$$
B\left(\sigma\left(B\left(t_{n} ; f\left(x_{n}\right), \varepsilon^{0}\right)\right), \phi\left(\varepsilon^{0}\right)\right)
$$

and thus $\pi\left(t_{n}, y_{n}\right)$ belongs to (8.21) for $n \geq \max \left(n^{*}, m^{*}\right)$. This contradicts (8.9).

Corollary 8.2. If $B, C, D, E \in \mathcal{P}(X), B \subseteq C, D \subseteq E, f: C \rightarrow E$ is a homeomorphism, $f(B)=D, B$ (and then also $D$ ) is compact, $\phi \in \Phi^{0}$, and $\pi^{B}$ and $\sigma^{D}$ are uniformly semi-stable, then

$$
\begin{aligned}
\left\{\left(\pi^{B}, C\right) \text { and }\left(\sigma^{D}, E\right) \text { are } f-\mathrm{M}(\phi) \mathrm{AE}\right\} & \\
& \Leftrightarrow\left\{\left(\pi^{B}, C\right) \text { and }\left(\sigma^{D}, E\right) \text { are } f-\mathrm{M}(\phi) \mathrm{UAE}\right\} .
\end{aligned}
$$

R e m a r k 8.7. If all assumptions of Theorem 8.1 are satisfied, except possibly the condition $\phi \in \Phi^{0}$, then one can prove the implication: if $\pi^{B} \mathrm{M}(\phi) \mathrm{AE}_{C} \sigma^{f(B)}$, then $\pi^{B} \mathrm{M}(\psi) \mathrm{UAE}_{C} \sigma^{f(B)}$ for every $\psi \in \Phi^{0}$ such 
that $\phi(\varepsilon) \leq \psi(\varepsilon)$. This applies for instance to $\psi$ defined by the formula $(*)$ in Remark 8.4.

Theorem 8.2. Let $D, G \in \mathcal{P}(X), \phi \in \Phi$ and $f: G \rightarrow X$ be given. Assume that $D \subseteq G, \sigma^{f(D)}$ is uniformly semi-stable in $X$ and

$$
\pi^{D} \mathrm{M}(\phi) \mathrm{UAE}_{G} \sigma^{f(D)} .
$$

Then $\pi^{D}$ is uniformly semi-stable in $G$.

Proof. Assume the contrary. So there is $\varepsilon^{0}>0$ such that for every $\delta>0$ and each $T \geq 0$ there are $x \in D$ and $t \geq T$ such that

$$
\varrho(\pi(t, x), \pi(t, y)) \geq \varepsilon^{0}
$$

for some $y \in B(x, \delta) \cap G$.

Using classical arguments we find sequences $\left\{x_{n}\right\}$ and $\left\{y_{n}\right\}$ in $X$ and $\left\{t_{n}\right\}$ in $\mathbb{R}$ such that for every $n$,

$$
x_{n} \in D, \quad y_{n} \in G, \quad t_{n} \geq n, \quad \varrho\left(x_{n}, y_{n}\right)<1 / n,
$$

but

$$
\varrho\left(\pi\left(t_{n}, x_{n}\right), \pi\left(t_{n}, y_{n}\right)\right) \geq \varepsilon^{0} .
$$

Uniform semi-stability of $\sigma^{f(D)}$ in $X$ implies existence of $\gamma>0$ and $T^{0} \geq 0$ such that

$$
\begin{aligned}
& \{u \in f(D), w \in X, \varrho(u, w)<\gamma\} \\
& \quad \Rightarrow\left\{\varrho(\sigma(t, u), \sigma(t, w))<\varepsilon^{0} / 3 \text { for } t \geq T^{0}\right\} .
\end{aligned}
$$

Take now $\varepsilon^{\prime} \in(0, \gamma)$ such that $\phi\left(\varepsilon^{\prime}\right)<\varepsilon^{0} / 6$. By (8.12) we can find $\delta^{\prime}>0$ and $T^{\prime} \geq 0$ such that for every $x \in D$,

$$
\pi_{t}\left(B\left(x, \delta^{\prime}\right) \cap G\right) \subseteq B\left(\sigma_{t}\left(B\left(f(x), \varepsilon^{\prime}\right)\right), \phi\left(\varepsilon^{\prime}\right)\right) \quad \text { for } t \geq T^{\prime} .
$$

For $n$ sufficiently large, say for $n \geq n^{*}$, we have

$$
\varrho\left(x_{n}, y_{n}\right)<\delta^{\prime} \quad \text { and } \quad t_{n} \geq \max \left(T^{0}, T^{\prime}\right) \text {. }
$$

The inclusion (8.26) holds true in particular for $t=t_{n}$ and $x=x_{n}$ if $n \geq n^{*}$, and so we have

$$
\pi\left(t_{n}, x_{n}\right) \in B\left(\sigma\left(t_{n} ; B\left(f\left(x_{n}\right), \varepsilon^{\prime}\right)\right), \phi\left(\varepsilon^{\prime}\right)\right)
$$

and simultaneously (compare the first condition in (8.27))

$$
\pi\left(t_{n}, y_{n}\right) \in B\left(\sigma\left(t_{n} ; B\left(f\left(x_{n}\right), \varepsilon^{\prime}\right)\right), \phi\left(\varepsilon^{\prime}\right)\right) .
$$

The implication (8.25) gives in particular (because $\varepsilon^{\prime}<\gamma$ )

$$
d\left[\sigma_{t}\left(B\left(f(x), \varepsilon^{\prime}\right)\right)\right] \leq 2 \varepsilon^{0} / 3
$$

for every $t \geq T^{0}$ and $x \in D$, and so, since $\phi\left(\varepsilon^{\prime}\right)<\varepsilon^{0} / 6$, we finally get for $t \geq \max \left(T^{0}, T^{\prime}\right)$

$$
d\left[B\left(\sigma_{t}\left(B\left(f(x), \varepsilon^{\prime}\right)\right), \phi\left(\varepsilon^{\prime}\right)\right)\right] \leq 2 \varepsilon^{0} / 3+2 \phi\left(\varepsilon^{\prime}\right)<\varepsilon^{0} .
$$


This is true in particular for $t=t_{n}, x=x_{n}$ if $n \geq n^{*}$. So for $n \geq n^{*}$,

$$
\varrho\left(\pi\left(t_{n}, x_{n}\right), \pi\left(t_{n}, y_{n}\right)\right) \leq \varepsilon^{0},
$$

which contradicts (8.24)

Corollary 8.3. If $D, G, E, F \in \mathcal{P}(X), D \subseteq G, E \subseteq F, \phi \in \Phi, f: G \rightarrow$ $F$ is a homeomorphism, $f(D)=E,\left(\pi^{D}, G\right)$ and $\left(\sigma^{E}, F\right)$ are $f-\mathrm{M}(\phi) \mathrm{UAE}$, then

$\left\{\pi^{D}\right.$ is uniformly semi-stable in $\left.G\right\}$

$$
\Leftrightarrow\left\{\sigma^{E} \text { is uniformly semi-stable in } F\right\} \text {. }
$$

Remark 8.8. All definitions and all results presented above have their natural modified versions concerning positive and negative semi-stability and approaching properties in pseudo-dynamical and dynamical systems.

\section{References}

[1] N. P. Bhatia and G. P. Szegö, Stability Theory of Dynamical Systems, Springer, Berlin, 1970.

[2] A. Pelczar, Semi-stability of motions and regular dependence of limit sets on points in general semi-systems, Ann. Polon. Math. 42 (1983), 263-282.

[3] - Limit sets and prolongations in generalized (multivalued) semi-systems, preprint WS-363, Vrije Universiteit Amsterdam, Faculteit Wiskunde en Informatica, 1990.

[4] - A contribution to the theory of generalized semi-systems: asymptotic equivalence and generalized prolongational limit sets, to appear.

[5] J. Sabine de Lis, An elementary explicit example of unbounded limit behaviour on the plane, Rev. Acad. Canaria Cienc. 5 (1) (1993), 41-46.

[6] A. Trzepizur, L'équivalence asymptotique au sens de Ważewski: un analogue d'un théorème de Levinson, Bull. Polish Acad. Sci. Math. 36 (1988), 39-46.

[7] T. Ważewski, Sur la coïncidence asymptotique des intégrales de deux systèmes d'équations différentielles, Bull. Acad. Polon. Sci. Lettres Sér. A Sci. Math. 1949, $147-150$.

INSTITUTE OF MATHEMATICS

JAGIELLONIAN UNIVERSITY

REYMONTA 4

30-059 KRAKÓW, POLAND 